\title{
Philosophiques
}

\section{Le regard d'ailleurs : les constructions utopiques de la « différence »}

\section{Daphne Patai}

Volume 21, numéro 2, automne 1994

Les femmes et la société nouvelle

URI : https://id.erudit.org/iderudit/027291ar

DOI : https://doi.org/10.7202/027291ar

Aller au sommaire du numéro

Éditeur(s)

Société de philosophie du Québec

ISSN

0316-2923 (imprimé)

1492-1391 (numérique)

Découvrir la revue

Citer ce document

Patai, D. (1994). Le regard d'ailleurs : les constructions utopiques de la " différence ». Philosophiques, 21(2), 525-545. https://doi.org/10.7202/027291ar
Résumé de l'article

L'évidence du concept de la " différence " entre hommes et femmes imprègne le discours féministe contemporain. Pour aborder ce concept de façon critique, j'utilise un corpus littéraire qui nous aide à voir l'évident, le non-remarqué et l'habituel dans nos positions sexuales : les fictions utopiques en forme d'inversion des rôles sexuaux. Après une discussion détaillée de ces textes, je suggère que la vision utopique de l'écrivaine anglaise Katharine Burdekin pointe en direction d'un sentier qui pourrait nous mener hors des interminables débats sur l'« essentialisme » et le « constructivisme social » comme explications de la « différence ». 


\title{
C O N F ÉRE R C E D E C I O I U R E
}

\section{Le regard d'ailleurs : les constructions utopiques de la « différence »}

\author{
par \\ Daphne Patai
}

\begin{abstract}
RESUME : L'évidence du concept de la « différence » entre hommes et femmes imprègne le discours féministe contemporain. Pour aborder ce concept de façon critique, jutilise un corpus littéraire qui nous aide à voir l'évident, le non-remarqué et l'habituel dans nos positions sexuales : les fictions utopiques en forme d'inversion des rôles sexuaux. Après une discussion détaillée de ces textes, je suggère que la vision utopique de l'écrivaine anglaise Katharine Burdekin pointe en direction d'un sentier qui pourrait nous mener hors des interminables débats sur l'« essentialisme » et le « constructivisme social » comme explications de la «différence».
\end{abstract}

ABSTRACT : The obviousness of the concept of « difference » between men and women permeates contemporary feminist discourse, which has devoted much energy to explicating the sources of this « difference ». In order to take a critical look at this concept, I draw on a body of literature that helps us to see the obvious, the unnoticed, the habitual in our gendered positions : utopian fictions cast as sex-role reversals. After discussing these texts in some detail, I argue that the utopian vision of the british writer Katharine Burdekin hints at a path that can lead us out of the endless debates over « essentialism » and « social constructionism » as explanations of « difference ».

Ce texte a pour objet l'évident, et la façon dont les choses que l'on considère évidentes glissent doucement dans l'invisibilité. Je soutiendrai que c'est précisément l'évident qui doit être rendu visible, et qu'afin de récupérer l'évident nous devons trouver un point de vue, un lieu d'où l'évident perd son évidence, est délesté de l'attribut du « cela va sans dire ». 
Dans un important article intitule « Archimedes and the Paradox of Feminist Criticism » (I98I), Myra Jehlen assimilait la quête féministe d'un lieu à partir duquel exercer son scepticisme radical, à la quête, par Archimède, d'un point d'appui pour soulever le monde. A l'inverse d'Archimède, dont la quête était vouée à l'échec par la nécessité de trouver « un point d'appui complètement en dehors de ce monde ${ }^{\mathrm{I}}$, les féministes, selon Jehlen, ont besoin d'un «point d'appui terrestre » qui les situerait « d'emblée dans le monde et hors du monde » (p. 576). D'après Jehlen, les programmes d'Études féministes ont simplement créé des enclaves incapables du moindre impact sur les disciplines et discours masculins, qui ont continué à constituer la norme. Par suite, le problème des chercheures féministes était de surmonter leur « base différente » (p. 577), c'est-à-dire leur isolement. Jehlen suggérait une issue à ce dilemme, du moins dans l'arène de la critique littéraire : l'adoption d'une méthode radicalement comparative, centrée sur la contradiction.

Mon propos est, à l'inverse, que nous disposons déjà d'un point d'appui archimédien virtuel grâce à certains textes de fiction - les spéculations utopiques - et que nous devrions nous en servir, les « expériences mentales » déclenchées par la fiction utopique offrant aux lectrices et lecteurs l'expérience imaginaire d'une réalité différente. Chercher un nouveau «lieu » dans le monde actuel, où la plupart des lieux sont déjà surdéterminés, c'est peut-être minimiser le rôle important de l'imagination dans la formation d'un sens du possible. En d'autres termes, l'expérience de lire une utopie et de s'immerger dans ses constructions sociales différentes situe effectivement lectrices et lecteurs, même si c'est de façon temporaire, dans un « ailleurs » qui nous offre une perspective unique d'où regarder et évaluer les structures d'évidence invisibles de notre monde. A cause de leurs éventuelles conséquences sur notre appréhension de la vie quotidienne, ces expériences imaginaires ont une grande importance, car le glissement qu'elles permettent dans notre perception de l'évident peut à la fois restaurer notre capacité à être étonné-e-s, et renouveler notre résolution d'agir.

L'évidence du concept de la « différence » entre femmes et hommes, voilà ce que je veux remettre en question dans cet essai. Ce concept imprègne le discours féministe contemporain. S'il a subi divers traitements lexicaux, il est toujours demeuré au cœur du débat féministe. Dans la théorie juridique, dans la critique littéraire, dans les analyses politiques, philosophiques et même biologiques de l'identité sexuale (gender), nos catégories fondamentales n'ont pas changé. Nous demandons : faut-il mettre l'accent sur l'« identité » ou sur la « différence »? Dans le domaine de la loi, les conséquences de l'adoption de

I. Myra Jehlen, « Archimedes and the Paradox of Feminist Criticism », Signs : Journal of Women in Culture and Society 6. n' 9, Eté 1981, p. 575-60I. 
l'une ou l'autre de ces catégories sont immédiatement visibles. Nier la différence en soulignant l'identité, cela peut, par exemple, conduire les femmes à renoncer au soutien de l'ex-mari en cas de divorce. Mais accepter la différence peut conduire à un renforcement de la division sexuale traditionnelle de la réalité : l'accent mis sur la différence peut, par exemple, justifier l'exclusion des femmes de certains emplois, et/ou stimuler des conditions d'emploi protectionnistes plutôt qu'égalitaires ${ }^{2}$. En un certain sens, la critique littéraire rejoue cette discussion : dénoncer le double standard et le caractère réactionnaire de la dévaluation masculine traditionnelle des écrivaines peut facilement conduire à un argument « égalitariste » (ou du moins à un argument de « la valeur comparable »), c'est-à-dire à l'argument que les femmes peuvent s'intégrer à la norme, que les écrivaines sont aussi bonnes que les écrivains, que l'échec à reconnaître leur valeur égale est surtout le résultat d'un simple préjugé, d'intérêts acquis, du fait de privilégier les modes et sujets masculins, de paradigmes canoniques stupides, et ainsi de suite. Et une telle prise de position encourt évidemment les mêmes risques que l'argument légal d'« identité » ou de « différence »: il ne réussit pas à contester la structure même de la situation préexistante, et se contente modestement de l'espoir d'y intégrer les femmes ${ }^{3}$. A l'encontre de tout cela, l'argument de la « différence » conteste l'idée que « se joindre à eux » soit une stratégie appropriée ou un but désirable, et souligne plutôt que les femmes écrivent différemment, doivent

2. Carol Smart écrit que, depuis le dix-neuvième siècle, la question fondamentale de la politique féministe a été de savoir « si l'Etat et la loi devraient accorder aux femmes un traitement spécial en vertu de leurs capacités spécifiquement féminines et de leurs supposées caractéristiques, ou si la justice serait mieux servie en traitant les femmes comme égales aux hommes, avec des droits et responsabilités égaux ». Voir son ouvrage Feminism and the Power of Law, Londres, Routledge, 1989, p. 82. Deborah L. Rhode, dans son article « The Woman's Point of View », Joumal of Legal Education 38, 1988, p. 39, note: « Durant la plus grande partie de l'histoire de l'Amérique, l'accent mis sur la perspective spécifique des femmes a joué à l'encontre des intérêts spécifiques des femmes ». Ainsi, par exemple, «la supposée différence dans l'aptitude des deux sexes au travail légal » a été utilisée pour empêcher les femmes d'entrer dans la profession juridique. Voir aussi Mary Jane Mossman, « Feminism and Legal Method : The Difference It Makes », Winconsin Women's Law Journal 3, 1987, P. 147-68; Susan Gluck Mezey, « When Should Difference Make a Difference : A New Approach to the Constitutionality of Gender-Based Laws », Women \& Politics Io, n' 2. 1990, p. 105-19; Martha Minow, Making All the Difference : Inclusion, Exclusion and American Law, Ithaca, N.Y., Cornell University Press, I990.

3. Dans Feminism and the Power of Law, p. 82. Carol Smart cite des théoriciennes comme Catherine Mackinnon, M. Thornton, et S.J. Kenney, qui ont montré que « ces deux approches présupposent que les hommes sont la norme qui sert à mesurer les femmes en tant que différentes ou les femmes en tant qu'égales. C'est la capacité reproductive des femmes qui crée un problème pour la norme masculine inhérente 
être jugées différemment, et qu'il ne s'agit pas de donner plus d'extension aux paradigmes masculins mais de les rejeter totalement ${ }^{4}$.

Ce qui, aujourd'hui, donne forme et signification à l'ensemble du débat critique, c'est avant tout le fait qu'il s'agit d'un assaut politique, d'un effort pour rendre visible la nature politique des jugements antérieurs qui ont réussi à se camoufler sous le manteau de la neutralité, comme des choses en soi. Bien entendu, cette attaque est motivée par le désir de faire éclater un discours particulier qui se considère comme l'état « naturel » des jugements adéquats. Inutile, ici, de ressasser les arguments familiers sur la nécessité d'étendre l'ancien canon, de créer un nouveau canon, ou de se dispenser de tout canon. Je veux plutôt attirer l'attention sur l'évident. Le groupe qui détient le pouvoir n'a jamais besoin de déclencher cette sorte de discussion. Tout ce qu'il a à faire, c'est de tenter de maintenir sa position centrale et son pouvoir en résistant aux attaques, en faisant dévier les questions, et en empêchant les redéfinitions. En fait, même sans beaucoup de résistance active, son pouvoir passé est son allié le plus efficace pour maintenir sa position, car l'exercice traditionnel du pouvoir est rarement perçu comme tel; on l'expérimente plutôt comme la façon dont sont les choses ${ }^{5}$. Ses définitions déterminent ce qui est légitime (dans la vie, la loi, l'art), et rien n'est plus simple que de démontrer que les contestations de ces définitions découlent de l'intérêt personnel. En effet, et cela n'est pas surprenant, ce sont les « étrangers » qui ont le plus besoin de déclencher ces

inhérente à la loi, et non, par exemple, la renonciation de l'homme à s'occuper des enfants. Dans cette perspective, ni l'approche différentielle ni l'approche égalitaire ne commencent même à effleurer le problème du pouvoir qu'a la loi de proclamer sa neutralité. Fondamentalement, ces approches laissent la loi telle qu'elle est, cherchant à trouver la manière la plus efficace de faire passer les intérêts des femmes par-delà les législateurs et le pouvoir judiciaire ».

4. La défense de la différence a été poussée à l'extrême par des féministes comme Hélène Cixous, qui mettent l'accent sur le corps - comme s'il y avait une relation nécessaire entre le flot menstruel et le flot créateur. Qu'on me permette aussi de préciser que, dans l'optique de la présente discussion, je néglige le problème important de la différence parmi, et non seulement entre, les groupes humains. Pour une analyse de plusieurs emplois contemporains du terme «différence », voir Michele Barrett, «The Concept of Difference », Feminist Review 26, Eté 1987, p. 29-4I.

5. Le langage même quej'utilise dans cet article est une illustration du problème que je tente de cerner : il s'agit (dans les termes de la division conventionnelle de la réalité en modes stéréotypés masculins et féminins) d'un langage androcentrique enraciné dans les métaphores militaires de l'assaut et de l'attaque, de la rébellion et du combat. Plus loin dans le texte, je citerai un passage de Katharine Burdekin suggérant un sentier différent - mais qui, sous plusieurs rapports, est également travaillé par le fait que notre langage même, s'il doit servir à la communication. ne peut à aucun moment aller trop loin au-delà des frontières de la convention. 
attaques. La pensée féministe n'a décidément pas résolu ce problème dans le secteur de la critique littéraire, ni, d'ailleurs, dans aucun autre secteur ${ }^{6}$.

Le vocabulaire, les glissements et déplacements dans ce qui est maintenant devenu le très long débat sur la différence des femmes ont été analysés en détail dans un article récent d'Ann Snitow ${ }^{7}$. Snitow fait référence à « la grande ligne de partage sexuale », ou à « la ligne de partage féministe »c'est-à-dire à la façon qu'ont les arguments sur la différence et l'identité de réapparaître continuellement, sous divers masques, comme des processus de pensée féministes, et de réapparaître non seulement entre des positions divergentes, mais aussi à l'intérieur de positions spécifiques, comme le montrait déjà l'ouvrage de Mary Wollstonecraft publié dès i792. Comme le souligne Snitow, la terminologie de la «minimalisation » et de la «maximalisation » proposée par Catharine Stimpson était une version précoce, dans la seconde vague du féminisme, de cette ligne de partage dans les discussions théoriques sur l'identité sexuale : devons-nous minimiser ou maximiser la différence entre les femmes et les hommes ${ }^{8}$ ? Plus récemment, nous avons utilisé à peu près dans le même sens l'opposition entre l'essentialisme et le constructivisme social. Égalité et différence, déclare Snitow, voilà le nom « le plus ancien, et certainement le plus compréhensif », de cette ligne de partage (p. 213-15), où la théorie de la différence tend à mettre l'accent sur le corps (et l'inconscient), alors que la théorie de l'égalité supprime l'accent sur le corps au profit d'un moi

6. Pour un exemple dans le domaine de la loi, voir Regina Graycar, « Equal Rights versus Father's Rights : The Child Custody Debate in Australia », dans Child Custody and the Politics of Gender. Carol Smart and Selma Sevenhuijsen (ed.), Londres et New York, Routledge, 1989, p. 158-189. Comme le souligne Graycar, la notion d'« égalité »-dans le cas présent, «des droits pour les parents », ce qui veut dire la garde partagée des enfants en cas de divorce (p. I83) - implique en fait la continuation du contrôle patriarcal après le divorce. Analysant les termes du débat, elle note qu'ils « reposent lourdement sur l'idéologie de l'égalité en même temps qu'ils ignorent la réalité de choses comme les pratiques quotidiennes du soin donné aux enfants par les mères et pères divorcés, et la situation économique interdépendante des hommes et des femmes en Australie » (p. 185). Sa thèse est que la rhétorique de la réalité ne devrait pas empêcher une analyse minutieuse des « réalités sous-jacentes » aux conventions de garde. Si nous ne sommes pas prêtes à entreprendre de telles analyses, l'argument « égalitaire », incrusté dans le cadre légal patriarcal traditionnel, peut tout simplement devenir un moyen de confirmer ce cadre. Pour une discussion comparable de la loi canadienne, voir Anne Marie Delorey, « Joint Legal Custody : A Reversion to Patriarchal Power », dans Canadian Joumal of Women and the Law 3, n' I, 1989, p. 33-44.

7. Ann Snitow, «Pages from a Gender Diary », Dissent, Printemps I989, p. 205-223. Une autre version de cet article figure dans Adrienne Harris and Ynestra King (eds), Rocking the Ship of State : Toward a Feminist Peace Politics, Boulder, Westview Press, I989. P. 35-73.

8. Catharine R. Stimpson, « The New Scholarship About Women : The State of the Art », Annals of Scholarship I, n², 1980, p. 2-14. 
non circonscrit par l'identité sexuale (p. 213). L'argument de Snitow, c'est que les théoriciennes féministes « ne cessent pas de rebaptiser cette tension, comme si de nouveaux noms pouvaient faire avancer le travail politique féministe »; elle nous prévient que les noms nouveaux peuvent nous faire oublier le nombre de fois que nous avons baptisé cette scission auparavant. J'ajouterais que cette activité de nommer et de renommer ne nous fait guère progresser dans la résolution d'un problème fondamentalement politique. Quelle que soit la terminologie, les lignes d'allégeance politiques, en fait, se rompent, car parmi les maximalistes, les essentialistes ou les adeptes de la différence, nous trouvons non seulement des féministes qui veulent insister sur la différence tout en se contentant d'inverser les étiquettes évaluatives (elles attachent un signe positif à la féminité, et un signe négatif à la masculinité), mais aussi des hommes anti-féministes utilisant à peu près les mêmes arguments pour perpétuer leur propre position privilégiée. Proclamer la «différence »-comme on le verra plus clairement plus loin - est un jeu que n'importe qui peut jouer, et pour les fins les plus diverses. Cela laisse tout à fait intact le problème ultérieur de savoir quoi faire avec cette différence, ou ce qui est présupposé par une telle proclamation.

Le point majeur à noter ici, c'est que toutes ces discussions sur la différence s'enracinent elles-mêmes dans la disparité politique. Cela veut dire qu'il est impossible ne serait-ce que de nommer la « différence » sans impliquer une comparaison et sans privilégier par inadvertance le terme non marqué de la comparaison. La différence, autrement dit, est un problème pour le groupe qui porte sa marque; elle n'est pas un problème pour le groupe non marqué qui est considéré comme la norme. Dans notre contexte, la différence est un problème pour les femmes, mais c'est un problème qui a été créé pour les femmes par les hommes. Je crois que c'est la signification de la fameuse phrase de Simone de Beauvoir : « On ne nait pas femme ». Au lieu de nous engager de façon non critique dans les arguments sur la différence, qu'il s'agisse de la célébrer, de la déplorer, ou de la déclarer un malentendu, nous devons remarquer comment toute la discussion est formulée et structurée. Ce détail apparemment trivial - que le débat soit simplement formulé de cette façon particulière - signale l'existence relative du pouvoir d'un côté et de l'impuissance de l'autre. C'est pourquoi, dans notre monde, ce ne sont pas les hommes qui ont intérêt à contester l'idée de la « différence » des hommes; ce ne sont pas les hommes qui doivent démontrer aux femmes leur identité; et ce ne sont pas les hommes qui s'engagent dans une auto-définition élaborée, toujours par rapport à un autre d'un statut plus élevé. Bien au contraire, ce sont les hommes qui sont le plus susceptibles d'adhérer à la notion de différence, naturalisant et neutralisant par là ce qu'autrement ils pourraient devoir confronter comme une position de domination maintenue à l'encontre des principes politiques mêmes que plusieurs d'entre eux (au moins dans notre société supposément démocratique) défendent. Dans Une chambre à soi, Virginia Woolf notait que les bibliotheqques étaient remplies de livres sur les femmes, mais que « les 
hommes » étaient beaucoup moins souvent leur sujet. Cela ne signifie pas, bien entendu, qu'il y a plus d'intérêt pour les femmes que pour les hommes, ou que les ouvrages consacrés aux femmes ont joui de plus d'importance. Au contraire, ce que révèle la remarque de Woolf, c'est que les hommes étaient (et sont) la norme, et que cela rend problématique l'existence des « femmes », qui se transforment alors en sujet de recherche, «sujet » en un double sens. Tandis que les femmes deviennent le sexe, le problème (Que veulent donc les femmes? demandait Freud avec une perplexité faussement candide, comme s'il s'agissait d'une autre espèce), dont le statut et l'être même requièrent d'interminables discussions et négociations, tout ce que les hommes ont à faire c'est ce qu'ils font d'habitude : la réalité conforte déjà leur position de toutes les façons possibles.

On peut obtenir une meilleure compréhension du processus en utilisant les intuitions de l'ethnométhodologie. Dans les termes de Dean MacCannell :

Dans le fonctionnement actuel de la vie sociale, chaque chose semble fermement attachée à sa signification... La façon dont la réalité ordinaire est structurée demeure intuitivement évidente. Le monde social est simplement saturé de signification, et d'une manière qui n'attire pas l'attention sur elle parce qu'elle est en train de devenir signifiante. C'est sa caractéristique la plus mystérieuse et la plus sociale ${ }^{9}$.

Dans le cas des inégalités sexuales, qu'on les considère comme enracinées dans les différences biologiques inhérentes ou dans les habitudes sociales, cela devient beaucoup moins important dès que l'on se concentre non sur la « différence » mais sur le pouvoir. Car c'est toujours et seulement le groupe qui regarde de l'extérieur, le nez contre la vitre, dont les caractéristiques et le statut subissent un examen détaillé. C'est ce groupe qui est « différent », et qui doit cajoler, convaincre et donner des raisons pour sa prétention à un changement de statut. Et, précisément à cause de leur impuissance habituelle et de la supposition qu'ils devraient être invisibles et inaudibles, les étrangers sont affectés de ce que j'appellerais une « visibilité supplémentaire », en vertu de laquelle tout espace qu'ils occupent, toute voix qu'ils élèvent, semblent excessifs et injustifiés. L'absence de pouvoir - qui a une capacité remarquable à se légitimer et à se neutraliser - a le don de faire apparaître ses constestations comme des cas particuliers, et partant faciles à délégitimiser ${ }^{10}$.

9. Dean MacCannell, The Tourist : A New Theory of the Leisure Class, New York, Schocken Books, 1976, p. II8.

Io. De mon point de vue, la visibilité supplémentaire offre une définition fonctionnelle du statut de minorité. Ce concept explique pouquoi toute action « répréhensible » de n'importe quel membre d'un groupe «minoritaire » - les noirs, les juifs, les femmes, les homosexuels - est considérée comme une caractéristique de l'ensemble du groupe, renforçant ainsi les stéréotypes; cette situation n'existe tout simplement pas pour le groupe dominant. Les membres des 
Dans notre monde, la différence, répétons-le, est un problème pour les femmes, pas pour les hommes. Dans la publicité télévisée, seules les femmes doivent déclarer, avec un sourire touchant de fierté et de conviction : «Parce que je le mérite ! » Il serait ridicule pour un homme d'énoncer un tel propos; les mots eux-mêmes, dans la bouche d'un homme, sembleraient un oxymore évoquant une image comique d'impuissance et de manque de confiance, car pourquoi dire ce que la plupart des gens croient et qui est confirmé par les interactions sociales de tous les jours ? En ce sens, la « différence » des femmes comprise comme une infériorité accablante - est pathétiquement incorporée même (ou particulièrement) aux bruyantes déclarations en sens contraire.

Semblablement, alors même que nous discutons la nature de la différence féminine, nous ne pouvons nous empêcher de reproduire le problème même que nous tentons d'esquiver. Car le fait demeure que nous sommes vraiment situées de différentes manières par la réalité courante, et nos efforts pour transformer la situation sont souvent dérisoires, comparés à la puissance insondable de « Ce Qui Est ». Dans cette perspective, il est important de passer d'un vocabulaire des « rôles » sexuaux à un vocabulaire des «positions » sexuales. Comme Bronwyn Davies et Rom Harré le soulignent, le mot « rôle » est habituellement incrusté dans les perspectives traditionnelles qui considèrent les positions sexuales comme statiques et fixes. Mais le discours (sans parler de la vie politique et sociale) situe en fait les individus, par l'entremise des interactions quotidiennes, dans certaines positions, et cela selon des manières particulières qui doivent être analysées et contestées ${ }^{\text {II }}$. Donc, que nous argumentions pour l'égalité ou la différence, nous devons remarquer que

groupes minoritaires sont sans doute intuitivement conscients de ce danger; c'est pourquoi leurs propres actions policières, ajoutées à celle du groupe dominant, maintiennent leur statut marginal. Les contestations de la norme, ou des programmes scolaires traditionnels, offrent des exemples significatifs du phénomène de visibilité supplémentaire au cours des récentes années. Cela explique les réponses de ceux qui, défendant le statu quo, exagèrent la menace de la « gauche », comme si, dès que «n'importe lequel d'entre eux » met le pied dans la porte, « tout le lot » semble entrer.

II. Bronwyn Davies and Rom Harré, « Positioning : The Discursive Production of Selves », Joumal for the Theory of Social Behaviour 20, n I, 1990, p. 43-63. Davies et Harré opposent leur conception dynamique et interactive de la « position » en tant que produite dans l'interaction discursive, au concept plus ancien de « rôle », qui sert à mettre de l'avant les aspects statiques, formels et rituels. J'ajouterais que la notion de « rôle » implique aussi - paradoxalement - quelque chose dont on peut se débarasser simplement en « changeant de rôle », ce qui camoufle effectivement les mécanismes élaborés par lesquels une « réalité »donnée est maintenue en place. Le concept de « position » permet une meilleure conception des processus sociaux complexes et entremêlés. Pour une analyse analogue de l'identité sexuale comme quelque chose qui se produit dans le fonctionnement des interactions quotidiennes, voir Candace West et Don H. Zimmerman, « Doing Gender », Gender \& Society I, n" 2 (June 1987). p. 125-51. 
ce sont les hommes (et leur pouvoir, qui inclut le pouvoir de définir la réalité, de reproduire leurs perspectives, et d'imposer ces perspectives comme si elles étaient neutres) qui constituent toujours le point de référence. Comment, dès lors, pouvons-nous sortir de ce cadre?

Je voudrais suggérer que le premier pas vers l'évasion consiste à considérer ce cadre précisément pour ce qu'il est : un cadre particulier, maintenu en place par des pratiques sexuales routinières et un discours traditionnel sexualisé. Le mouvement des femmes savait cela il y a une décennie ou deux - quand l'idée de l'homme comme norme perdit son évidence et s'étala comme l'expression idéologique du pouvoir qu'elle est en fait. Cette récupération de l'évident était extrêmement importante pour les femmes, en tant qu'action à la fois intellectuelle et politique. Mais dans les discussions de plus en plus sophistiquées de la différence qui ont eu lieu depuis lors, cette simple perception politique a à nouveau eu tendance à se perdre. Ainsi, le féminisme s'est trop souvent donné corps et âme à la discussion de la différence - comme si cette discussion valait d'être poursuivie pour elle-même - sans se rappeler que les implications politiques de la «différence » sont ce qui la rend digne d'être discutée.

Il existe tout un corpus littéraire qui nous aide à percevoir l'évident, le non-remarqué, l'habituel, dans nos positions sexuales. Il s'agit des fantaisies utopiques d'inversion des rôles sexuaux ${ }^{12}$. Par un simple glissement de perspective, ces inversions rendent le fonctionnement du pouvoir instantanément visible. Dans le monde de l'inversion des rôles sexuaux, la hiérarchie prévaut mais ce sont les femmes qui dominent. Ainsi, la « différence » devient un problème pour les hommes, et ce sont les hommes qui doivent se débattre pour obtenir des gains politiques en arguant que leur « différence » ne devrait pas conduire à l'impuissance, ce sont les hommes qui avancent des arguments à l'encontre des thèses féminines concernant la biologie et la socialisation des hommes, la nature et la culture. Ce sont les hommes qui se préoccupent anxieusement de chaque mot proféré par les femmes dont dépendent leur subsistance et leur bien-être, leur langage corporel révélant leur statut inférieur; ce sont les hommes qui se plaignent d'être délaissés, de ne pas être pris au sérieux, de leurs possibilités d'emploi limitées et de leurs tâches domestiques routinières et ennuyeuses. Et ce sont les hommes qui se fatiguent des pratiques sexuelles égocentriques des femmes et de leurs arguments humiliants à propos de l'infériorité biologique masculine, ce sont les hommes dont la sexualité et l'image corporelle sont réprimées et objet de distorsions. Vivre

12. Je reviens ici à la terminologie des « rôles sexuaux » parce que l'expression « inversion des rôles sexuaux » est devenue une appellation classique dans les bibliographies et études sur la fiction utopique. La technique littéraire de l'inversion des rôles sexuaux est elle-même ancienne : elle était déjà à l'œuvre dans L'assemblée des femmes d' Aristophane. 
imaginairement, pour quelques heures ou quelques jours, dans ces sociétés où le pouvoir sexual est inversé, cela a un effet étonnant : on en revient avec la conscience soudaine que, du point de vue de ces sociétés imaginaires, c'est notre propre monde qui repose sur une bizarre inversion des rôles sexuaux, une inversion où les hommes dominent, et que nous prenons cela pour la « réalité $»^{13}$.

13. Je veux cependant établir une distinction entre les inversions de rôles sexuaux soigneusement machinées des romans que je vais discuter, et l'usage plus limité de l'« estrangement » sexual dans les représentations du travestissement vestimentaire. Dans le travestissement vestimentaire ce n'est pas le pouvoir sexual qui est inversé, mais simplement le pouvoir personnel, qui s'ajoute ou s'efface grâce à un déguisement efficace qui vous fait passer pour un membre du sexe opposé. Un film comme Tootsie offre un bon exemple du genre de contradictions qui abondent dans le changement de position sexuale, habituellement limité, des arts populaires. Alors qu'il se propose de critiquer les stéréotypes sexuaux, le film, ultimement, les confirme. Avec le personnage de Dorothée, le film nous présente une admirable figure féminine - crâne, dure et... masculine. Dorothée, telle qu'interprétée par un Dustin Hoffman travesti, est une femme meilleure, au sens de plus admirable, et certainement plus efficace, que les vraies femmes du film. Mais il n'y a rien de neuf dans le message qu'un homme réussit mieux - quel que soit l'objet de sa réussite. Dans Tootsie, les scènes où Dustin Hoffman en tant qu'homme, tempête et fulmine devant son co-locataire contre la façon dont il a été (mal)traité en tant que femme constituent les seuls moments du film qui commencent à produire l'« estrangement » grâce auquel les meilleures utopies d'inversion des rôles sexuaux se développent. Mais la principale distinction à faire entre les romans et le film, à part le problème du double standard idéologique, c'est que les inversions de rôles sexuaux remettent toute une société sur ses pieds; elles ne traitent pas l'oppression sexuale comme un problème purement individuel qui peut être réglé par une personne qui a recours au travestissement vestimentaire dans une société intacte. Les spectatrices et spectateurs peuvent quitter Tootsie en s'émerveillant de l'étrangeté de Dustin Hoffman, en vêtements de femme, qui se fait tapoter le derrière, mais toujours sans considérer outrageux le fait de voir Jessica Lange recevoir le même traitement. Ou, pis encore, l'habillement féminin peut rendre si familier le comportement qu'il permet que nous pouvons éviter de nous étonner de voir le personnage d'Hoffman, Dorothée. traité de façon sexiste de manière routinière, et ne ressentir le sens d'un affront que la scène est censée évoquer, que quand on voit à nouveau Hoffman en homme. Dans une telle situation, nos émotions peuvent être trop absorbées par un personnage, dont le sort seul semble compter, et le potentiel politique du travestissement est annulé. Dans le film Tootsie, une telle interprétation est renforcée par la scène finale, où Dustin Hoffman, maintenant vêtu d'un habillement masculin débraillé qui aurait vivement embarrassé Dorothée, exerce son magnétisme masculin sur une Jessica Lange réceptive, quoique hésitante, et delicieusement séduisante. 
Voilà pourquoi je considère l'argument de la différence comme un jeu que les deux camps ${ }^{14}$ peuvent jouer, un jeu dont la signification découle entièrement non de détails biologiques, mais d'un cadre politique donné. Aucun argument de ce genre ne peut nous dire quoi que ce soit à propos de ce que «sont » les hommes ou les femmes, il ne peut nous permettre de voir que ce que nous savons déjà : que, dans une société donnée, certains groupes occupent certaines cases qui, simultanément, les invitent et les contraignent à perpétuer la réalité dominante; et que des contestations de cette domination peuvent advenir et adviendront de façon passablement prévisible, et rencontreront des obstacles prévisibles.

On ne défait pas facilement des inégalités de pouvoir grâce à des actes de contestation verbale. Les arguments mêmes que nous employons à propos du pouvoir, comme les arguments à propos de l'identité sexuale, qui en dérivent de façon évidente, se produisent toujours dans un contexte où le pôle dévalorisé se trouve déjà sur la défensive ${ }^{15}$. C'est pourquoi les discussions sur l'infériorité des femmes s'accompagnent de présupposés sur la supériorité des hommes. Les hommes n'ont pas besoin de se demander si leur pouvoir réside dans ce qu'ils sont ou dans ce qu'ils font - même s'ils sont prêts à répondre à de telles questions en cas de contestation. Mais les femmes doivent entamer cette discussion - ce qui, inévitablement, les place dans une position désavantageuse. Les inversions de rôles sexuaux mettent en évidence ces manœuvres. Dans les discours théoriques, il nous faudrait recourir à une multiplicité de textes différents pour découvrir précisément comment le langage, les manières d'élever les enfants, la posture corporelle, les vêtements, les mœurs, l'éducation, les croyances religieuses, les codes légaux, les routines

14. Utiliser une terminologie comme « chaque côté », c'est encore une fois trop concéder à la réalité dominante et à ses métaphores oppositionnelles. Comme le souligne Dorothy Sayers dans une inversion pleine d'esprit, « The Human-NotQuite-Human », publiée dans son livre Unpopular Opinions : Twenty-One Essays, New York, Harcourt, Brace, 1947, p. 142-49, il est étrange que l'on désigne les femmes comme « le sexe opposé ». «Quel est, s'étonne Sayers, le « sexe voisin »?» (p. 142). La question « innocente » est un instrument très important d'« estrangement ».

15. Dans son article « Repressive Tolerance », dans Robert Paul Wolff, Barrinton Moore Jr., et Herbert Marcuse, A Critique of Pure Tolerance, Boston, Beacon Press, 1965. p. 92, Herbert Marcuse fait référence à « l'inégalité instituée » de notre société : « La struture antagonique de la société détermine les règles du jeu. Ceux qui s'opposent au système établi sont a priori désavantagés, et cet inconvénient n'est pas supprimé par la tolérance de leurs idées, discours et journaux ». La « tolérance » accordée à ces idées marginalisées n'altère pas leur statut marginal. Qu'on puisse les énoncer et les négliger contribue à l'apparence de libre et égale compétition des idées et perspectives. L'adoption d'une perspective politique, par opposition à un point de vue abstraitement éthique, permet à Marcuse de percevoir les fonctions « répressives » d'une telle tolérance. 
domestiques, les pratiques sexuelles - et toutes les nombreuses autres façons par lesquelles la domination d'un groupe sur un autre se traduit dans les différents aspects de la vie quotidienne - à la fois soutiennent l'inégalité sexuale et l'affichent. Mais n'importe quelle histoire relativement simple d'inversion des rôles sexuaux révélera plusieurs des mécanismes interreliés de domination sexuale, et cela même à un lecteur ou à une lectrice plutôt non avertis. Tel est le pouvoir de l'acte de mettre l'accent sur le non-familier, lequel ramène soudainement l'évident en pleine lumière.

Il n'a d'ailleurs pas fallu attendre les discussions de la fin du vingtième siècle pour suggérer de telles inversions aux écrivaines féministes. Il y a plus d'une centaine d'années, Annie Denton Cridge, dans son court récit Man's Rights, or How Would You Like It? $(\mathrm{1} 870)^{16}$, a imaginé une société matriarcale dans laquelle ce sont les hommes qui sont obsédés par leur apparence, qui sont banalisés et excédés de travail. Les hommes opprimés décrits par Cridge sont privés de droits politiques à l'aide d'arguments parfaitement plausibles tirés de la nature, comme celui qu'avance une femme élégante et sûre d'elle-même s'opposant aux demandes des hommes concernant leurs droits civils et politiques : «En outre, comme la Nature connaît bien la supériorité de la femme et l'infériorité de l'homme, elle qui a dévolu la maternité à la femme ! » Et puisque la Nature est harmonie, la supériorité physique de la femme se reflète aussi bien dans sa supériorité mentale. Par suite, il est logique que les hommes prennent en charge toutes les tâches d'entretien ménager et de soins des enfants qui ne sont pas immédiatement liées à l'enfantement, à la fois pour compenser leur infériorité biologique et pour soulager le fardeau de leur conjointe de toute évidence supérieure à eux. Comme plusieurs autres écrivaines féministes œuvrant dans ce genre, Cridge empêche ses lectrices et lecteurs de voir là une simple satire, car elle laisse sa narratrice (une rêveuse de notre monde) faire le commentaire suivant: « Je pensais dans mon rêve que je n'étais pas du tout satisfaite du propos de la dame, et j'éprouvais vraiment de la pitié pour ces pauvres petits messieurs juchés sur l'estrade et contraints d'entendre tant de choses sur leur infériorité » (p.3r). Parce qu'elle se rend compte que ce ne sont pas seulement les rôles privés des femmes qui doivent changer, mais aussi leurs rôles publics, Cridge fait jouer l'inversion et sur la scène du foyer individuel, et sur la place publique, ce qui lui permet d'offrir à ses lectrices et lecteurs une perspective distanciée sur leur propre réalité, par l'entremise de la réaction des femmes au pouvoir au spectacle particulier

I6. Annie Denton Cridge, Man's Rights, or, How Would You Like It?, Wellesley. Mass., Mrs. E.M.F. Denton, I87o. Pour une discussion détaillée de cette précoce histoire d'inversion des rôles sexuaux, et de plusieurs autres récits du même genre, voir mon article « When Women Rule : Defamiliarization in the Sex-Role Reversal Utopia », Extrapolation 23, n" I. Printemps 1982, p. 56-69. 
$\mathrm{d}^{\prime}$ hommes réclamant un espace public ${ }^{17}$. De cette façon, Cridge remet nos perceptions habituelles sur leurs pieds. Quand, à la fin du livre, sa narratrice dit à ses hôtes que, dans son propre monde, ce sont les hommes qui règnent, ses propos sont accueillis avec cette sorte d'incrédulité qui exprime la conviction jamais remise en question que la façon dont les choses sont est la seule possible.

Plus d'une centaine d'années plus tard, l'écrivaine norvégienne Gerd Brantenberg écrivit Egalia's Daughters, originellement publié en Norvège en 1977 et traduit en anglais en 1985 . Les brillants arguments de Brantenberg concernant l'infériorité biologique masculine sont présentés de façon si convaincante dans ce roman que lectrices et lecteurs en seront peut-être conduits à se demander comment les femmes ont jamais pu être embobinées jusqu'à croire le contraire - ce qui, bien entendu, est la chose à saisir ${ }^{18}$.

En Egalia, le pays imaginaire de Brantenberg, la notion familière que la biologie est un destin est pulvérisée précisément par le renversement intégral des rôles sexuaux et de tous les multiples signes du pouvoir qui caractérisent notre société patriarcale. Le langage est l'élément clef de ce renversement, alors que Brantenberg nous introduit dans un monde où les fams dominent et accomplissent le travail qui importe dans le monde - en tant que fams d'affaires, fams de mer, fams de pêche et ainsi de suite. Les « Mères Formatrices » constituent une pierre de touche idéologique, et des expressions comme « la race hufam », «par Lucie! », et « eh bien, je serais une fille de chien! » abondent. La poétesse nationale d'Egalia est Walta Whitman; et il y a un mouvement sparksiste, fondé sur l'analyse de la société de classes proposée par une certaine Clara Sparks ${ }^{19}$. Presque tout ce qui caractérise et définit la société patriarcale occidentale a un analogue inversé dans ce tour de force d'imagination. Les zomfams opprimés portent des « pehos »- des soutien-

17. Ann Snitow, op. cit., p. 214, cite les propos d'Ellen Dubois : « Mon hypothèse est que la signification du mouvement pour le suffrage des femmes repose précisément sur le fait qu'il négligeait l'oppression des femmes dans la famille, ou la sphère privée, et réclamait plutôt leur accession à la citoyenneté et, grâce à celleci, à l'arène publique ». Voir l'article de Dubois, « The Radicalism of the Woman Suffrage Movement : Notes Toward the Reconstruction of Nineteenth-Century Feminism », dans Anne Phillips, (ed.), Feminism and Equality. New York, New York University Press, 1987.

18. En fait, des arguments similaires avaient été avancés des décennies plus tôt dans le livте spirituel et incisif de Ruth Herschberger Adam's Rib, New York, Pellegrini \& Cudahy, 1948; réimprimé par Harper $\&$ Row, 1970. Voir en particulier le chapitre huit, «La société écrit la biologie ». La recherche féministe n’a pas encore résolu le problème de l'oubli réitéré de ce qu'on devrait considérer comme des textes clefs et qu'il faut, semble-t-il, constamment ressusciter.

19. Gerd Brantenberg, Egalia's Daughters : A Satire of the Sexes, Trad. par Louis Mackay avec la coopération de Gerd Brantenberg, Seattle, The Seal Press, 1985. Brantenberg m'a écrit (dans une lettre datée du 26 février 1987) qu'elle a joué seule avec cette idée 
pénis - par analogie avec le mot norvégien pour soutien-gorge, «bristholder 》, qui est rarement prononcé à haute voix mais d'habitude raccourci en $\mathrm{BH}$, un euphémisme, explique Brantenberg ${ }^{20}$, qui n'est pas trop embarrassant. Même si un tel scénario a eu des précédents - en particulier en ce qui a trait au code vestimentaire, au comportement et au pouvoir social (matière première du répertoire de l'inversion des rôles sexuaux) - c'est dans son traitement du comportement sexuel que Brantenberg conteste le plus efficacement les arguments tirés de la biologie.

L'idéologie sexuale d'Egalia est, comme la nôtre, fondée sur la notion que l'anatomie est le destin : ainsi est-ce la biologie masculine qui rend les zomfams d'Egalia inférieurs aux fams dirigeantes, qui relègue les zomfams au statut de rois du foyer, anxieux d'obtenir (de leurs partenaires sexuelles, ces fams qui les exploitent et les dominent) la protection due à la paternité. Inversant complètement l'argument que la femme est à la nature comme l'homme est à la culture, la société matriarcale de Brantenberg souscrit à la conclusion profondément raisonnable que, dans un monde où existe une telle opposition entre nature et culture, ce sont les hommes qui sont au plus près de la nature, et donc moins précieux pour la civilisation, car c'est seulement chez les hommes que plaisir sexuel et procréation sont intimement liés : l'orgasme

pendant plusieurs années, et qu'elle n'aurait probablement pas pris la peine d'écrire le livre si elle avait été au courant de l'existence d'autres inversions de ce genre. Les problèmes de traduction, déclare-t-elle, étaient énormes :

«Vous devez le repenser dans chaque nouvelle langue. Comme j'ai passé une bonne partie de ma vie à tenter d'apprendre l'anglais (comme langue seconde), j'ai collaboré à la traduction du livre dans cette langue...

[...] "Mères formatrices" (Moulding Mothers) est bien sûr une parodie des "Pères fondateurs" (Founding Fathers), lesquels n'ont existé nulle part ailleurs qu'aux EtatsUnis en I776. Mais nous avons chez nous "les Pères d'Eidsvoll" de I8I4, année où nous avons obtenu notre indépendance (du Danemark) comme nation; en norvégien, j'ai donc fait une version parodique de cela - les Mères au mont Demo... Beaucoup de mots/phrases/idées se perdent dans la traduction anglaise, parce qu'en norvégien il y a en général plus de termes à référence sexuale qu'en anglais... Certains mots, cepandant, sont des gains. Comme "ladsel". Des "ladsel" en détresse. "Lordies and gentlewim" est une expression comique sans équivalent dans le texte norvégien.

Clara Sparks, au lieu de Karl Marx, c'était un élément d'invention métonymique rendu possible par la familiarité de Brantenberg avec l'Angleterre; dans ce pays, une chaîne de grands magasins appelée "Marks and Spencer" est nommée familièrement "Mark and Sparks". Dans le texte norvégien, le personnage s'appelle Gulfuria, qui n'est "qu'un nom inventé à la consonance amusante". »

20. Brantenberg, lettre du 26 février i987. Egalia's Daughters peint aussi le développement d'un mouvement de zomfams, et d'un mouvement gai au sein des zomfams. Pour les besoins de cet article, je m'en suis cependant tenue à la représentation qu'offre le roman des relations de pouvoir entre hommes et femmes. 
accompagne l'éjaculation ${ }^{21}$. D'après cette conception, la supériorité des femmes, en termes de développement évolutionnaire, est manifeste dans leur séparation caractéristique de la procréation (via l'acte sexuel) et du plaisir sexuel (orgasme). Mais, en fait, cette reconstitution d'un argument biologique en faveur de l'infériorité « naturelle » déconstruit complètement la biologie en tant que catégorie pertinente à la détermination du pouvoir sexual, car rien n'est plus simple pour Brantenberg que de démontrer avec une logique implacable le fait que non seulement les rôles sexuaux mais aussi le comportement sexuel au niveau le plus intime sont des constructions sociales. A ma connaissance, son roman est le seul à conduire l'inversion directement dans le dernier bastion de la pensée biologique : la chambre à coucher.

En Egalia, donc, ce sont les zomfams qui doivent prouver qu'ils prennent leurs pilules anticonceptionnelles, et qui doivent obtenir une permission spéciale s'ils veulent devenir pères; ce sont les fams qui utilisent les hommes pour leur propre plaisir sexuel (se frottant vigoureusement contre la cuisse du zomfam indolent, recourant à la masturbation manuelle en agrippant son pénis, etc.); ce sont les fams qui imposent leurs exigences sexuelles à des zomfams qui préfèreraient dormir; ce sont les zomfams qui n'ont pas eu d'orgasme depuis des siècles, et qui n'entrent même que rarement en érection. Ce dernier point est particulièrement intéressant : puisque les fams ont décrété que l'érection masculine n'est pas nécessaire à leur plaisir, et puisque la tradition de la domination des fams empêche les zomfams de formuler (et même de trouver le langage pour le faire) leurs propres désirs sexuels, les « faits » biologiques de la société patriarcale - c'est-à-dire l'érection et une constante disponibilité sexuelle (la prétendue norme masculine de notre société) - cessent d'en être en Egalia. Au lieu de cela, l'élément clef mis en lumière par la fiction de Brantenberg n'est rien d'autre que la question du pouvoir fondé sur la domination : qui domine? qui est en position d'utiliser qui ?

En Egalia, la conception instrumentale de l'homme constitue la norme: les zomfams sont, sur le plan sexuel, le jouet des fams, qui les molestent, abusent d'eux et les violent, puis blâment la victime d'avoir été provocante, ou de s'être trouvée au mauvais endroit au mauvais moment. Les zomfams, tout à fait sensibles au pouvoir féminin, sont à la merci des fams; par suite, les fams les plus agées qui ont réussi et qui ont du pouvoir sont «naturellement » considérées attirantes par plusieurs zomfams jeunes et ambitieux; et, si l'attrait sexuel du pouvoir échoue, le harcèlement et le pouvoir économique peuvent toujours prendre la relève. Si un zomfam féconde une fam qui ne veut

21. Ruth Herschberger, dans Adam's Rib, p. 74, fait la même remarque que Brantenberg mais, et cela est intéressant, dans un contexte radicalement différent. Discutant l'idée que, pour les femmes, « l'orgasme est un luxe », elle cite The Physiology of Sex. de Kenneth Walker : « Alors que, pour l'accomplissement satisfaisant de la fonction masculine de fertilisation l'éjaculation, et donc l'orgasme, est indispensable. pour la fonction féminine de conception l'orgasme n'est pas nécessaire ». 
pas lui donner la protection de paternité, c'est lui qui est disgracié, et c'est aux paroles de la fam qu'on accorde créance (à moins, évidemment, qu'il ne puisse prouver qu'il a dûment pris sa pilule anticonceptionnelle - ce qu'on fait en public une fois par mois). C'est lui qui doit s'inquiéter de ses moyens d'existence et craindre l'abandon. Dans une telle société, le fait que ce sont les femmes, et non les hommes, qui donnent naissance aux enfants constitue une source supplémentaire et une preuve du pouvoir des femmes et de l'impuissance des hommes. « Naturellement », l'enfantement est un acte révéré et récompensé (et dissocié du soin ultérieur des enfants), tandis que le milieu de travail est entièrement adapté aux besoins et habitudes des fams ${ }^{22}$.

Brantenberg ne conteste pas les faits biologiques fondamentaux. Elle ne « libère » pas les femmes de leur capacité reproductive spécifique; plutôt, elle se rend compte que la signification sociale de la grossesse est le vrai problème, et que celui-ci est liéà la position inférieure des femmes dans la société patriarcale non en tant que cause, mais en tant que raison d'être. Ce ne sont pas toutes les histoires d'inversion des rôles sexuaux qui considèrent comme inévitables les rôles biologiques du mâle et de la femelle dans la reproduction. Mais ce qui offre un intérêt particulier, dans les textes qui le font, ce sont précisément les arguments tout à fait raisonnables et logiques que l'on peut construire pour justifier une inversion des rôles politiques et des rapports de domination, en se fondant sur les « faits » biologiques familiers. Ai-je besoin de préciser que ces écrivaines ne présentent pas leur vision comme une utopie authentique c'est-à-dire ne suggèrent pas que la domination en tant qu'instrument politique doit exister - mais offrent plutôt un portrait satirique de leur propre société, avec l'intention d'inciter leurs lectrices et lecteurs à remettre en question des catégories qui sont normalement acceptées et prises pour acquises, et qu'elles font cela en recourant largement à l'humour? Brantenberg elle-même a commenté ce fait : « [L'] idée est très simple, et elle a tout simplement surgi, parce que tous les arguments en faveur du maintien du patriarcat sont stupides. Et même si la stupidité peut mener à la cruauté et à la tragédie, elle n'en est pas moins risible ${ }^{23}$.

22. On trouvera dans le roman The New Gulliver, or, The Adventures of Lemuel Gulliver, Jr. in Capovolta, New York, Taplinger, 1979. d'Esmé Dodderidge, une étude semblable, mais particulièrement détaillée, de ce à quoi ressembleraient les rôles sur le marché du travail et au foyer si les femmes, et non les hommes, étaient le sexe dominant, et si les autres valeurs de la société moderne demeuraient intactes.

23. Lettre du 9 avril 1987 . Dans la même lettre. Brantenberg déclare aussi que la première idée d'un tel livre lui est venue au début des années 1960 : « Mais écrire un livre requiert une certaine expérience et une certaine maturité - une certaine connaissance de la façon dont fonctionne la société, des aperçus que je n'avais pas encore quand j'ai eu pour la première fois l'idée d'écrire ce livre alors que j'avais environ vingt ans; j'ai commencé à l'écrire, mais ne l'ai jamais terminé. Il était très différent alors. » Le livre a été écrit alors que Brantenberg, très active dans le mouvement des femmes danois, se trouvait à Copenhague. 
Ce ne sont pas seulement les écrivaines féministes comme Brantenberg qui décrivent le pénis comme « le membre le plus faible ». Même certain-e-s écrivain-e-s non féministes et anti-féministes d'histoires d'inversion des rôles sexuaux, dont les cuvres ont pour but ultime de réaffirmer la justesse ou l'inévitabilité de nos structures sexuales familières, en viennent à proposer d'excellents arguments biologiques en faveur de la supériorité politique et sociale des femmes. Par exemple, dans son roman satirique Regiment of Women (1973), Thomas Berger énonce l'argument gynocratique que l'infériorité politique des hommes est liée à leur physiologie, car ils ont des organes génitaux externes facilement vulnérables, ce qui ne peut manquer d'entraver leurs mouvements et leur capacité d'agir dans le monde ${ }^{24}$.

Pouvons-nous dépasser ces arguments sur la biologie et la société ? Ces manières de formuler le problème, comme le note Snitow dans l'article cité plus haut, continuent à hanter le cœur du féminisme; voilà pourquoi, par exemple, il n'y a pas de consensus féministe à propos de sujets aussi particuliers que les technologies de reproduction ou la pornographie. En réaction à la suggestion de Linda Alcoff, qui soutient que nous devons transcender le dilemme en développant une troisième voie, Snitow montre qu'aucune transcendance, aucune troisième voie n'est possible : « La contradiction pressante que les femmes expérimentent continuellement, entre les pressions pour être une femme et les pressions pour ne pas en être une, ne changeront que grâce à un processus historique; on ne peut les dissoudre par la seule pensée $\gg^{25}$.

Mais les histoires d'inversion des rôles sexuaux que j'ai décrites montrent clairement que ce problème n'en est pas un de terminologie ou de définition (que sont la « femme », l'« homme », la « différence »?) mais de pouvoir politique. Quand les hommes dominent, les femmes, comme le montre Snitow, sont prises au piège d'une constante oscillation entre l'identité et la différence comme position d'où formuler leurs demandes. Si les femmes étaient au pouvoir - comme elles le sont dans les sociétés différentes mises en œuvre par la technique fictionnelle de l'inversion des rôles sexuaux - ce serait l'« homme » et ce qu'il signifie qui seraient soumis à la même argumentation, aux mêmes scissions, lignes de partage et tensions. Pour s'apercevoir de cela, il faut prendre du recul par rapport à toute cette discussion. Pourquoi n'est-ce pas un problème urgent pour les hommes de notre société, de déterminer si « homme »

24. Thomas Berger, Regiment of Women, New York, Simon and Schuster, 1973. Le point culminant du roman, du moins en ce qui concerne les protagonistes, c'est la « rectification » de l'inversion, avec l'homme à nouveau au-dessus (littéralement). Des arguments biologiques familiers sont réitérés dans la dernière ligne du roman : « De plus, c'était lui qui possédait l'organe protubérant » (p. 349).

25. Snitow, p. 209. Ici, elle exprime explicitement son désaccord avec l'article de Linda Alcoff, «Cultural Feminism Versus post-Structuralism : The Identity Crisis in Feminist Theory », Signs 13, n I, Printemps 1988. 
a une signification plutôt sociale ou plutôt biologique? Parce que les hommes jouissent déjà d'une position privilégiée, et que seules les femmes, en les combattant, sont continuellement forcées de se remettre en question, de se redéfinir et de se resituer.

Dans un article antérieur intitulé « Beyond Defensiveness : Feminist Research Strategies $», j$ 'ai suggéré de prendre les femmes pour norme de notre discours, pour point de référence, et de procéder à partir de là ${ }^{26}$. À l'époque, j'étais frappée par les pièges d'une position éternellement combative et oppositionnelle (une position fatalement marquee par la condition de l'« Autre »). Mais je n'avais pas encore découvert, alors, les aperçus suggérés par l'œuvre d'une importante écrivaine anglaise de fiction utopique qui a soulevé à plusieurs reprises les mêmes problèmes. Katharine Burdekin (I896-ıg63) a publié dix romans, sous son propre nom ou sous le pseudonyme masculin « Murray Constantine », entre ig22 et 1940. Burdekin entrevoyait un futur tantôt masculiniste, tantôt féministe, et elle a écrit deux romans qui, sous ce rapport, se reflètent l'un l'autre. I'un est la dystopie Swastika Night écrite en 1936 et publiée pour la première fois en 1937 par «Murray Constantine $»^{27}$. Dans ce roman étonnant, qui décrit l'Europe 700 ans après la victoire du nationalsocialisme allemand, Burdekin découvre dans le « culte de la masculinité » l'élément clef du fascisme. La contrepartie de Swastika Night, composée, je crois, avant que l'attitude de plus en plus agressive d'Hitler n'oblige Burdekin à imaginer un avenir nazi, a pour titre The End of This Day's Business. Écrit autour de 1935, mais pas publié avant 1989, ce roman se situe 4000 ans dans le futur, à une époque oủ les femmes règnent sur le monde dans la paix et l'harmonie; le seul prix à payer pour leur règne, c'est la sujétion des hommes qui, ignorant leur propre histoire, et sans accès au langage du savoir, mènent une vie insignifiante dans leur plaisante sphère personnelle. Ne pouvant recourir à la terminologie de la fin du vingtième siècle de «l'objectivation sexuelle » et de la

26. Daphne Patai, « Beyond Defensiveness : Feminist Research Strategies », Women's Studies International Quaterly $6: 2,1983$, p. 67-89. Repris dans M. Barr and N.D. Smith (eds), Women and Utopia : Critical Interpretations, Lanham, MD., University Press of America, 1984; et dans Resa L. Dudovitz (ed.), Women in Academe, Oxford, Pergamon Press, 1984.

27. Murray Constantine, Swastika Night, London, Victor Gollancz, 1937; réédité comme sélection du club du livre de la gauche en ig40. Au milieu des années ig80, j'ai établi qui était l'auteure réelle du roman, et il a été réédité sous le nom de Burdekin à Londres par Lawrence et Wishart, et à New York par The Feminist Press. Voir mon introduction à cette réédition et mon article « Orwell's Despair, Burdekin's Hope : Gender and Power in Dystopia », Women's Studies International Forum 7, n 2. 1984, p. 85-96, ainsi que mon livre The Orwell Mystique : A Study in Male Ideology. Amherst, University of Massachusetts Press, 1984, chapitre 8. 
"mystique féminine », Burdekin a néanmoins décrit l'existence de ces phénomènes chez les hommes désarmés et sans pouvoir ${ }^{28}$.

Dans l'œuvre de Burdekin, des thèmes importants des derniers romans sont souvent préfigurés dans les plus anciens. L'inversion des rôles sexuaux de The End of This Day's Business a d'abord été évoquée dans un passage d'un récit antérieur, le roman pacifiste intitulé avec justesse Quiet Ways, publié en I930. Ici, pour un bref moment, Burdekin déploie sa vision d'une manière d'être différente, où la « femme » n'est pas une catégorie perpétuellement soumise à la diffamation, en quête d'analyse et de justification. Helga, l'héroïne de Burdekin, mesure six pieds et pèse i6o livres; elle est décrite comme si autonome que cela est intolérable du point de vue d'un misogyne conventionnel nommé, de façon appropriée, Carapace. La scène est racontée du point de vue de l'homme. Par ses réactions, lectrices et lecteurs en viennent à percevoir le travail sexual de routine accompli par les femmes, même quand elles tentent de contester le pouvoir masculin, et l'effet déstabilisant pour un homme du fait qu'une femme sorte de ce cadre simplement en s'abstenant de s'engager dans des actes routiniers de déférence ou de protestation. Burdekin oppose l'« homminisme » de Carapace (terme probablement emprunté à George Bernard Shaw) au « féminisme » d'Helga :

Carapace méprisait les femmes qui singeaient les hommes, mais cela au moins était flatteur. Cette impénétrabilité opaque lcelle d'Helgal, cette autosuffisance, cette absence presque méprisante d'attention quand les hommes parlaient étaient un présage. C'était nouveau et dangereux; si cela se généralisait, cela menaçait de perturber le monde beaucoup plus que le féminisme ordinaire, concentré sur le droit de vote et les professions. Carapace était un homministe passionné, et son foyer spirituel était la Grèce à l'époque de Périclès. Son âme sensible était à jamais piquée et écorchée par les idées égalitaires du vingtième siècle. Mais Helga faisait plus que le piquer et l'écorcher; elle le lacérait. Pour la première fois de sa vie, il sentait que la féminité n'était pas impertinente, faible, fondamentalement indécente et méprisable, mais une chose massive, patiente, immensément puissante. Il était profondément effrayé qu'Helga ne se croie pas inférieure à Alan Ison maril, et si des femmes comme elle devenaient la norme, pardieu. qu'adviendrait-il du monde? N'élèveraient-elles pas leurs filles en leur inculquant le même sens calme de la supériorité, non pas moins, mais plus dangereux parce qu'il ne s'affirmait pas bruyamment? N'élèveraient-elles pas leurs jeunes fils pour qu'ils croient leur valeur absolue moindre que celles des filles? Se projetant dans l'avenir, le cerveau de Carapace entrevoyait l'inversion complète de l'ordre normal des choses. Abolition de l'adoration du fils, remplacée par l'adoration des filles. Les femmes élevées de façon à remercier Dieu de n'être pas des hommes. Les garçons élevés de façon à souhaiter être des femmes. Il n'y avait pas de limites aux horreurs qui pouvaient se produire si les femmes perdaient un jour réellement le

28. Katharine Burdekin, The End of This Day's Business, afterword by Daphne Patai, New York. The Feminist Press, I989. Je suis tombée sur ce roman parmi plus d'une douzaine de manuscrits inédits de Burdekin; j'ai eu accès à ces manuscrits grâce à l'exécutrice littéraire de Burdekin, et à ses héritiers. 
sens de leur infériorité. Et qu'il pouvait être complètement absent, il en avait la preuve devant lui ${ }^{29}$.

Si j'ai cité ce passage intégralement, ce n'est pas par désir de promouvoir un quiétisme qui pourrait être (més)interprété comme un acquiescement à la réalité masculiniste. Plutôt, ce qui m'y frappe maintenant, alors que j'émerge des débats sur l'identité et la différence, c'est le sens rafraîchissant de l'espace transmis par la première partie de ce passage - son évocation d'une sorte de zone tampon intérieure où une femme se trouve authentiquement, même si c'est de façon momentanée, hors d'atteinte des interminables argumentations formulées entièrement en termes de pouvoir masculin et de perpétuel besoin, pour les femmes, de le contester. Le temps d'un éclair, Carapace sent la menace que pose à la réalité androcentrique une telle façon d'être. Entérinant comme il le fait l'inévitabilité de la hiérarchie, il saute d'emblée à la conclusion qu'une telle indépendance mènerait les femmes à une simple inversion qui modifierait la position des joueurs individuels, mais non les termes de la partie. Ce qu'il perçoit, en d'autres mots, c'est que le pouvoir masculin n'est pas un fait absolu et immuable, mais une relation qui dépend de la subordination des femmes. Dans un roman datant de 1934, intitulé Proud Man (publié sous pseudonyme), Burdekin ira plus loin en déclarant que les efforts révolutionnaires sont voués à l'échec s'il ne peuvent être plus que des « inversions de privilèges ${ }^{30}$. Elle donne vie à cet argument dans The End of This Day's Business, qui soulève le problème politique de la domination - cette fois par les femmes - dans un monde fort semblable à celui du cauchemar de Carapace.

Entretemps, nous qui connaissons les termes de notre situation dans le monde réel, nous devons réagir contre eux. Mais il me semble que si nos réactions demeurent captives des structures et concepts que le « patriarcat » met à notre disposition, nous gagnons peu de choses. Ne pouvons-nous pas croire avec un peu moins de véhémence en la réalité de concepts comme l'« essentialisme » et le « constructivisme social », pour nous réserver plutôt un espace où ces positions particulières ne constituent pas la somme intégrale de notre expérience ou de notre existence? Après tout, je n'expérimente pas ma « différence » tant qu'on ne me l'impose pas de l'extérieur. Tant que je continue à participer aux discussions habituelles de cette différence - qu'il s'agisse de tenter de la localiser, de la définir (ou redéfinir), de m'en excuser ou de la valoriser, je reproduis les structures mêmes quej'ai entrepris de contester. Il n'est certes pas requis de nous, et encore moins nous y contraint-on, que nous accordions pleine créance aux termes créés par notre marginalisation. Quelqu'un s'imagine-t-il sérieusement que, si nous devions un jour résoudre le problème des fondements adéquats d'une discussion sur le changement de

29. Katharine Burdekin, Quiet Ways, London, Thornton Butterworth, 1930, p. 239-40.

30. Murray Constantine, Proud Man, London, Boriswood, 1934. Roman récemment réédité par The Feminist Press (New York). 
statut des femmes, toutes les femmes s'y rallieraient, le consensus s'établirait, et le changement (tel que « nous » le concevons) se produirait nécessairement? Ou avons-nous simplement créé un autre secteur de discours - intéressant, cela va de soi - reposant sur le présupposé tacite que le bon discours, si on finit par tomber dessus, le bon discours bien développé, ou soutenu par une bonne argumentation, règlera le problème?

La vision venue d'ailleurs peut nous dessiller les yeux ${ }^{3 \mathrm{I}}$.

31. Note du traducteur (G.B.). Tout au long de ce texte, « gender » a été traduit par « identité sexuale », et ses dérivés par « sexual/sexuaux », adjectif qui désigne la différence entre les sexes sans connotation spécifiquement sexuelle ou biologique. On a préféré cette solution à l'utilisation de « genre », même si ce terme est de plus en plus fréquent dans la littérature féministe francophone, parce que cette utilisation, dès que l'on s'écarte du substantif, aboutit souvent à des contresens ( l'homme est un être générique ») ou à des balourdises (« l'homme est un être de genre »). Par ailleurs, les mots « fams » et «zoms » (dans «zomfams ») sont empruntés à L'Euguélionne de Louky Bersianik. 